\title{
Chromosomal abnormalities in recurrent spontaneous abortions: A retrospective study
}

\author{
Inusha Panigrahi ${ }^{1}$, Mohd. Shariq ${ }^{1}$, Ravi Thakur ${ }^{2}$, Subhas Saha ${ }^{1}$, and Gurjit Kaur ${ }^{2}$ \\ ${ }^{1}$ Post Graduate Institute of Medical Education and Research \\ ${ }^{2}$ Government Medical College and Hospital (GMCH), Chandigarh
}

November 25, 2020

\begin{abstract}
Purpose: Evaluation of recurrent spontaneous abortions (RSA) can be challenging for a Obstetrician. In case of early first trimester abortions, chromosomal abnormalities can be identified as an important cause. We analysed the RSA cases followed up and diagnosed in the Genetic Clinic or Genetic Lab of 2 hospitals in the region. Methods: Those couples with 3 or more spontaneous abortions were included in the analysis. Karyotyping was one using standard protocol with G-banding and reporting as per ISCN guidelines. Results: Of 97 RSA couples, 20 showed chromosomal abnormalities, and 15 of these had balanced chromosomal rearrangements. The age ranged from 22 years to 37 years, and the median number of abortions was 4. Complex chromosomal rearrangement was seen in 2 couples, in one partner. The spectrum of chromosomal anomalies in couples with RSA is discussed here. Conclusions: Frequency of chromosomal abnormalities in RSA was higher in present study compared to previous studies. Reciprocal translocations were commonest abnormality.
\end{abstract}

Sl.

No.

Age

(Year's)

No. of Abortion

(Trimester)

Types of Chromosomal Abnormalities in Couples

Types of Chromosomal Abnormalities in Couples

Types of Chromosomal Abnormalities in Couples

Female Partner

Male Partner

Male Partner

1.

$29 \mathrm{Y} / \mathrm{F}$

3 - First trimester losses

$46, \mathrm{XX}, \mathrm{t}(1 ; 10)(\mathrm{q} 41 ; \mathrm{q} 21)$ 
$46, \mathrm{XY}$

$46, \mathrm{XY}$

2.

$30 \mathrm{Y} / \mathrm{F}$

3 - First trimester losses

46,XX,t(1;3)(p22;q21)

$46, \mathrm{XY}$

$46, \mathrm{XY}$

3.

$30 \mathrm{Y} / \mathrm{F}$

3 - First trimester losses

46,XX,t(5;8)(q33;q11.2)

$46, \mathrm{XY}$

$46, \mathrm{XY}$

4.

$28 \mathrm{Y} / \mathrm{F}$

4 - First trimester losses

46,XX,t $(4 ; 5)(\mathrm{q} 28 ; \mathrm{p} 15)$

46,XX,t(4;5)(q28;p15)

46,XX,t $(4 ; 5)(\mathrm{q} 28 ; \mathrm{p} 15)$

5.

$30 \mathrm{Y} / \mathrm{F}$

4 - First trimester losses

46,XX,t(6;7)(p21.3;p13)

$46, \mathrm{XY}$

$46, \mathrm{XY}$

6.

$30 \mathrm{Y} / \mathrm{F}$

4 - First trimester losses

46, XX

46,XY,t(11;14)(q23;q31)

46,XY,t(11;14)(q23;q31)

7.

$29 \mathrm{Y} / \mathrm{F}$ 
5 - First trimester losses

46,XX,t $(4 ; 13)(\mathrm{p} 15.2 ; \mathrm{q} 12)$

$46, \mathrm{XY}$

$46, \mathrm{XY}$

8.

$30 \mathrm{Y} / \mathrm{F}$

5 - First trimester losses

46,XX,t(3;18)(q29;q21.1)

$46, \mathrm{XY}$

$46, \mathrm{XY}$

9.

$30 \mathrm{Y} / \mathrm{F}$

5-First \& 1-Second trimester losses

46, XX

46,XY,t(2;7)(q24;q31.2)

$46, X Y, \mathrm{t}(2 ; 7)(\mathrm{q} 24 ; \mathrm{q} 31.2)$

10.

$35 \mathrm{Y} / \mathrm{F}$

5 - First trimester losses

46,XX, t(15;19)(q14;q13;4)

$46, \mathrm{XY}$

$46, \mathrm{XY}$

11.

$28 \mathrm{Y} / \mathrm{F}$

5 - First trimester losses

46,XX,t(8;9;16)(q13;q13;q22)

46,XX,t(8;9;16)(q13;q13;q22)

$46, X Y$

12.

$38 \mathrm{Y} / \mathrm{F}$

8 - First trimester losses

46,XX,t $(1 ; 4 ; 3)(\mathrm{p} 13 ; \mathrm{q} 31 ; \mathrm{q} 21)$

46,XX,t(1;4;3)(p13;q31;q21)

$46, \mathrm{XY}$ 
13.

$28 \mathrm{Y} / \mathrm{F}$

4 - First trimester losses

46,XX,rob t $(13 ; 14)(\mathrm{q} 10 ; \mathrm{q} 10)$

46,XX,rob t(13;14)(q10;q10)

$46, \mathrm{XY}$

14.

$22 \mathrm{Y} / \mathrm{F}$

4- First trimester losses

45,X [60] / 47,XXX [16]

45,X [60] / 47,XXX [16]

$46, \mathrm{XY}$

15.

$25 \mathrm{Y} / \mathrm{F}$

4- First trimester losses

$46, \mathrm{XX}$

$46, \mathrm{XX}$

46,XY,inv $(9)(\mathrm{p} 11 ; \mathrm{q} 13)$

16.

$37 \mathrm{Y} / \mathrm{F}$

4- First trimester losses

46,XX,9qh+

$46, \mathrm{XX}, 9 \mathrm{qh}+$

$46, \mathrm{XY}$

17.

$26 \mathrm{Y} / \mathrm{F}$

3- First trimester losses

$46, \mathrm{XX}$

$46, \mathrm{XX}$

46,XY,9qh+

18.

$30 \mathrm{Y} / \mathrm{F}$

3- First trimester losses

46,XX 
$46, \mathrm{XX}$

46, XY,Yqh+,15ps+

19.

$30 \mathrm{Y} / \mathrm{F}$

3- First trimester losses

$46, \mathrm{XX}, 13 \mathrm{ps}+$

$46, \mathrm{XX}, 13 \mathrm{ps}+$

$46, \mathrm{XY}$

\section{Hosted file}

Table 2_RSA_Ms.pdf available at https://authorea.com/users/378829/articles/495236chromosomal-abnormalities-in-recurrent-spontaneous-abortions-a-retrospective-study

\section{Hosted file}

Ms_RSA_Chrome_FINAL_F3.pdf available at https://authorea.com/users/378829/articles/495236chromosomal-abnormalities-in-recurrent-spontaneous-abortions-a-retrospective-study

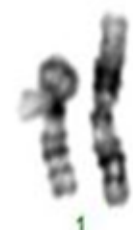

1

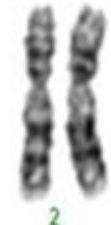

2

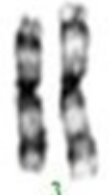

3

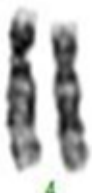

4

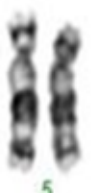

5

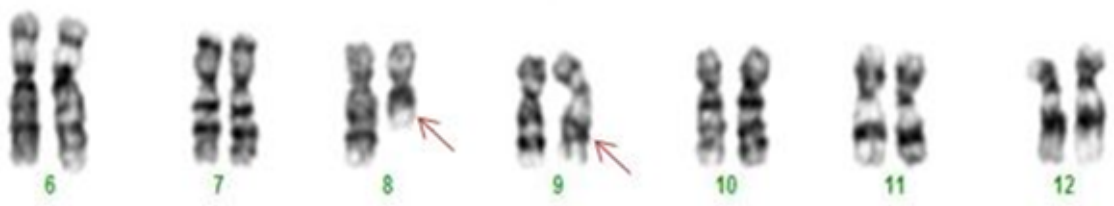

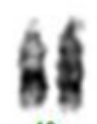

13

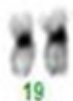

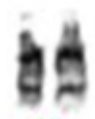

14

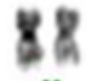

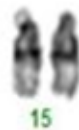

15

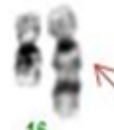

16
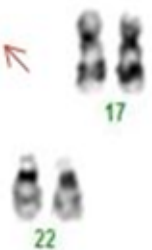

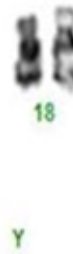

18

$\gamma$

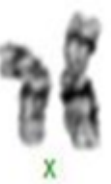



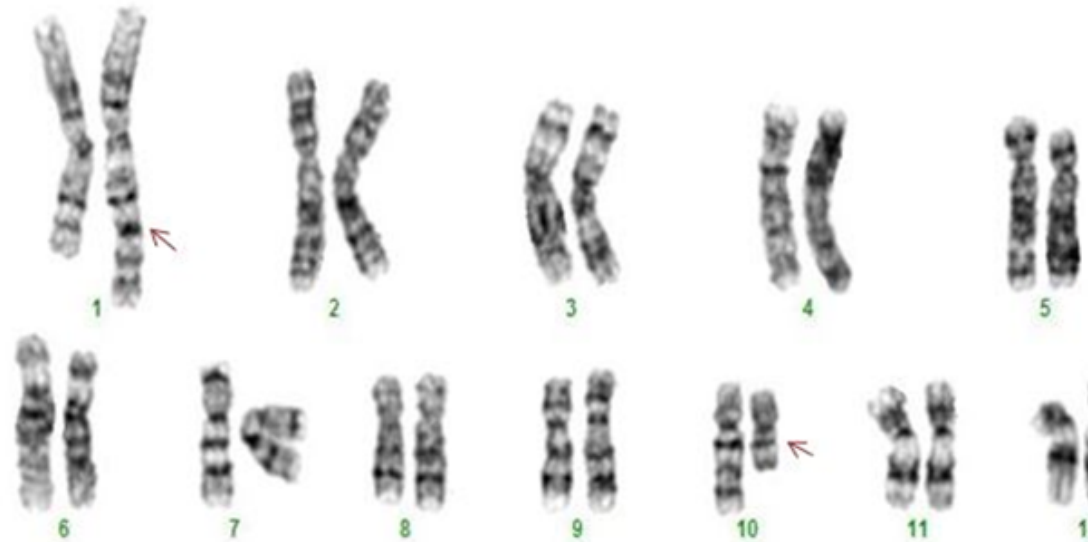

$\operatorname{lin}_{9}^{8}$

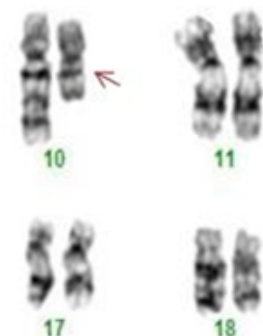

$7_{12}^{8}$

es

+6)

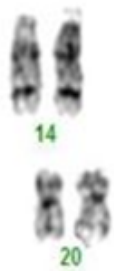

8.

8 을

(1)

${ }_{21}$

$\frac{8}{22}$

题点 